\title{
Acute Renal Failure During a Trial of Spinal Cord Stimulation: Theories as to a Possible Connection
}

Thomas M. Larkin, $\mathrm{MD}^{1,2}$, Anthony Dragovich, $\mathrm{MD}^{2}$, and Steven P. Cohen, $\mathrm{MD}^{3}$

From: ${ }^{1}$ Parkway Neuroscience and Spine Institute, Hagerstown, MD; ${ }^{2}$ Pain Management Center, Walter Reed Army Medical Center; ${ }^{3}$ Pain Management Center, Johns Hopkins Medical Institutions, Baltimore, MD, and Walter Reed Army Medical Center, Washington, DC.

Dr. Larkin is with the Department of Physiatry at Parkway Neuroscience and Spine Institute, Hagerstown, MD and the Department of Anesthesia and Operative Services, Pain Management Center, Walter

Reed Army Center. Dr. Dragovich is also with the Anesthesia and Operative Service,

Pain Management Center, Walter Reed Army Medical Center. Dr. Cohen is Associate

Professor, Department of Anesthesiology and Critical Care Medicine, Pain

Management Division, Johns Hopkins School of Medicine, Baltimore, MD; and Director of

Chronic Pain Research, Walter Reed Army Medical Center, Washington, DC

Address correspondence: Thomas M. Larkin, MD Parkway Neuroscience and Spine Institute 17 Western Maryland Parkway, Suite 100, Hagerstown, MD 21740 E-mail: larkintm@comcast.net

Disclaimer: There was no external funding in the preparation of this manuscript. Conflict of interest: None.

Manuscript received: 01/20/2008 Revised manuscript received: 07/03/2008

Accepted for publication: 07/08/2008

Free full manuscript: www.painphysicianjournal.
Objective: This is the first case describing an episode of acute renal failure occurring during a spinal cord stimulation trial.

Clinical Presentation: A 48-year-old male with a history of hypertension and 3 prior failed spine surgeries underwent a trial of spinal cord stimulation for uncontrolled bilateral lower extremity neuropathic pain. Two days after the placement of the percutaneous stimulator lead the patient returned complaining of 3 syncopal episodes. He was found to be hypotensive and in acute renal failure with a creatinine of 8.1 and a BUN of 83 .

Intervention: The stimulator lead was immediately removed. The patient was admitted to the intensive care unit and responded promptly to rehydration and placement of a urinary catheter. His renal and urological work-ups revealed no significant abnormalities.

Conclusion: The development of the episode of acute renal failure may have been influenced by the secondary effects of spinal cord stimulation. Since acute renal failure has never been associated with the use of spinal cord stimulation, this singular example does not by itself demonstrate a relationship. However, if future episodes are seen, a link between the 2 events could be drawn. For now, it is not clear if the development of this patient's acute renal failure could, in part, be attributed to the use of the spinal cord stimulator or if it was merely coincidental in nature. We do feel it is useful for the clinician to understand the pathophysiologic changes associated with spinal cord stimulation and to see how, at least in theory, there could be a connection.

Key words: acute renal failure, spinal cord stimulation

Pain Physician 2008; 11:5:681-686 ver the past 30 years, spinal cord stimulation (SCS) has become widely accepted in the medical community as a treatment for refractory, chronic pain conditions. Originally based on Melzack and Wall's "Gate Control Theory of Pain" (1), SCS was first described by Shealy et al (2) back in 1967 to treat a patient with intractable lower extremity pain. Since then, alternate theories have arisen to explain how SCS works to include activation of second order neurons and inhibitory interneurons at the level of the dorsal horn $(3,4)$, reducing the level of excitatory amino acids via increased gamma amino butyric acid 
levels (5), and release of endogenous opioids (6). The indications for SCS have also gradually expanded to pelvic and abdominal pain, malignant pain, brachial plexopathies, central pain, peripheral vascular disease, complex regional pain syndromes, angina, and failed back surgery syndrome (7-10). Whereas initial reports were tempered by high complication and failure rates, the advent of newer and more versatile systems have improved success rates and lowered complication rates (11-13).

Spinal cord stimulation is commonly used to treat nonmalignant, refractory pain that has failed to respond to conservative therapy. Among the various pain conditions that fall into this category, the most common one treated with SCS is failed back surgery syndrome (FBSS) (14). Turner et al (15) recently conducted a systematic review of SCS in patients with FBSS or complex regional pain syndrome). Although the best studies addressed CRPS I patients, there were enough relevant studies to conclude that SCS provides mild to moderate relief of pain in FBSS patients, with leg pain responding better than back pain. The mean complication rate across the 22 studies reviewed was $34.3 \%$, with the most frequently cited ones being the need for stimulator revision (23\%) or removal (11\%), equipment failure $(10 \%)$, pain in the region of hardware $(5.8 \%)$, and superficial infection (4.5\%). Less frequently reported complications include aberrant stimulation (16), refractory insomnia (17), atelectasis, pneumonia (18) and increased stimulation in the vicinity of a high-tension electricity substation (19). In this report, we describe a patient with FBSS who developed acute renal failure diagnosed 3 days after a spinal cord stimulator trial. To the best of our knowledge, this complication has never been previously reported. Possible mechanisms for this phenomenon are discussed.

\section{Case Report}

A 48-year-old male with a history of hypertension, gastroesophageal reflux, and hepatitis B was referred to the Walter Reed Army Medical Center pain management clinic for evaluation of persistent bilateral lower extremity pain status post-spine surgery. The patient had a complex history of low back and right leg pain dating back to 1979 after a fall aboard a naval ship. Since that time he had undergone extensive treatment ranging from medical management to surgery. His surgeries included a lumbar fusion at L5-S1 with 2 subsequent revisions. After his surgeries he was left with significant low back and leg pain that was poorly managed with medications and multiple spinal injections.

On initial evaluation, the patient described his low back pain as constant and sharp, with radiation into the right calf and left buttock. He had associated numbness and paresthesias, but denied weakness or bowel/bladder symptoms. His analgesic medications at that time included sustained-release morphine $200 \mathrm{mg}$ po BID, cyclobenzaprine $10 \mathrm{mg}$ po TID, and approximately $90 \mathrm{mg}$ per day of immediate-release morphine for breakthrough pain. His physical examination was remarkable for an intact sensory exam with normal motor strength, an absent left Achille's reflex, and a negative straight leg raise test. Baseline laboratories were not performed, but per patient report a complete blood cell count and full chemistry panel a little more than a year earlier were normal. On the visual analogue pain scale, he rated his pain as an $8 / 10$ at rest, increasing to $10 / 10$ with activity.

After discussing treatment options with the patient and his referring neurosurgeon, the decision was made to proceed with a spinal cord stimulator trial after removal of his bone growth stimulator. In October 2004, the patient had a single lead spinal cord stimulator placed percutaneously (Medtronic Verify® $60 \mathrm{~cm}$ lead) in the morning. The patient was on NPO status since the prior evening. During the trial no sedation was administered, although the patient did receive $500 \mathrm{ml}$ of lactated ringer's solution. His vital signs throughout the trial were notable only for a slightly elevated systolic blood pressure in the range of 150 $-160 \mathrm{~mm} \mathrm{Hg}$. The skin was entered from a paramedian approach at the L2-3 interspace to enable epidural entry at the L1-2 level. Placement of the lead itself took a single pass and was advanced until the tip reached the middle of the T9 vertebral body. The trial itself took approximately 40 minutes, after which the patient was discharged home with standard postimplant instructions.

Later that evening, the patient experienced a syncopal episode while walking to the bathroom, which was presaged by dizziness. His symptoms resolved after he laid down in bed. On POD \#1 he continued to feel dizzy and was unable to urinate. On POD \#2 he returned to the pain clinic requesting that the stimulator be removed. He could not recall urinating for the previous 2 days. Upon questioning, he stated that he continued to experience lightheadedness 
when upright, but that his symptoms resolved with recumbency. After removal of the spinal cord stimulator, the patient was sent to the emergency room for further evaluation. Initial vital signs in the ER were heart rate $88 / \mathrm{min}$, blood pressure $77 / 34 \mathrm{mmHg}$, respiratory rate $22 / \mathrm{min}$, temperature 98.6 degrees $\mathrm{F}$, and oxygen saturation $99 \%$ on room air. His electrocardiogram was normal. Laboratory data was as follows: $\mathrm{Na}+136, \mathrm{~K}+3.5, \mathrm{Cl}-95, \mathrm{HCO}-24$, blood urea nitrogen $83 \mathrm{mg} / \mathrm{dl}$, creatinine $8.1 \mathrm{mg} / \mathrm{dl}$, creatinine clearance of $16 \mathrm{ml} / \mathrm{min}$, glucose 102, CPK of $383, \mathrm{MB}$ of 1.7 , and troponins $<0.1$, with a normal complete blood count. After infusion of 3 liters of Ringer's lactate, his blood pressure increased to $100 / 56 \mathrm{mmHg}$, his heart rate declined to $73 / \mathrm{min}$, and he was able to produce a small amount of urine. He was subsequently transferred to the medical ICU where a renal ultrasound showed normal appearing kidneys and a urinalysis was normal. The following day his creatinine had decreased to $3.1 \mathrm{mg} / \mathrm{dl}$. By discharge, 2 days after admission, his creatinine decreased to $1.0 \mathrm{mg} /$ $\mathrm{dl}$, his blood urea nitrogen was $23 \mathrm{mg} / \mathrm{dl}$, and he was voiding spontaneously. Subsequent urological workup was remarkable only for mild obstruction secondary to prostatic hypertrophy and otherwise normal urodynamics.

\section{Discussion}

In this paper, we present a patient with hypertension and failed back surgery syndrome who developed acute renal failure (ARF) during a trial of SCS. While the facts are indisputable, what remains to be answered is what relationship, if any, SCS had on his development of ARF. There are no reports of spinal cord stimulators placed in any location, or for any length of time, precipitating renal failure or azotemia. From this patient's post-recovery laboratory values it is clear that he did not have underlying chronic renal insufficiency. He likely was markedly hypovolemic around the time of the SCS placement. This can be concluded from his electrolytes which show a normal bicarbonate level in the face of a highly elevated BUN which normally is consistent with uremia and a substantial acidosis. This either points to a pre-existing contraction alkalosis or a very rapid and significant renal correction which seems unlikely given his ARF. Laboratory error seems unlikely given the gradual reduction in his creatinine level with fluid resuscitation and the fact that he was oliguric at admission.
Our patient did have several independent factors that put him at risk for the development of ARF. These include some of the medications he was taking at the time of the SCS trial and his history of hypertension. The angiontensin-converting enzyme (ACE) inhibitor lisinopril, the thiazide diuretic hydrochlorothiazide, and high doses of morphine all can adversely effect renal function and volume status (20-22).

While in patients with certain types of nephropathies, ACE inhibitors have been shown to have renoprotective properties (23), paradoxically, in patients with congestive heart failure or severe hypovolemia, the use of an ACE inhibitor may actually precipitate acute renal failure (24). The reported patient had a history of hypertension which was modestly controlled at the time of the procedure. Hypertension itself can lead to a hypovolemic state that can be unmasked in the face of decreased sympathetic input. But it would seem unlikely that the stable use of an ACE inhibitor, a moderate and stable dose of morphine, and mild hypertension alone would lead to the dramatic changes noted in this case report.

Before embarking on a discussion of the effects of SCS on renal function one must identify the etiologic factors that lead to ARF. A recent study on the causes of ARF developing in intensive care unit patients identified the most common contributors to ARF as hypotension, sepsis, nephrotoxic drugs and volume depletion (25).

Any of these factors alone or in combination can lead to ischemic renal failure. The long-term prognosis of ARF is dependent on the speed at which the underlying etiologic factors are corrected. In order to achieve a rapid recovery as witnessed in this case, these factors normally need to be addressed between 2 to 12 hours (26). The patient's post-procedure creatinine level of $8.1 \mathrm{mg} / \mathrm{dl}$ suggests that this process may have been going on for a more prolonged period of time. However, a more chronic condition might not be expected to resolve so quickly and emphatically. Thus, the key question that arises from this case report is to what extent a precedent condition, volume depletion incurred by the surgery, and the surgery itself each contributed to the ensuing ARF.

SCS is widely acknowledged to affect autonomic equilibrium either via sympathetic inhibition or antidromal activation of sensory fibers (27-31). How exactly these alterations are achieved is not clear. There have been no studies to date evaluating the effects 
of lower thoracic SCS on renal blood flow. There are, however, a plethora of clinical and animal studies that show that SCS does indeed produce vasodilation via 2 mechanisms: a decrease in sympathetic outflow and a release of neurohumoral factors that directly lead to relaxation of (micro)vascular smooth muscles.

There is ample evidence that SCS can attenuate sympathetic responses. This idea was first postulated after it was noted that the effects of surgical sympathectomy mimicked the vasodilatory effects of SCS (32). The concept is further supported by animal studies indicating that the vasodilatory effects of SCS are reduced or obliterated by surgical sympathectomy (33). In animal studies evaluating the role cholinergic and adrenergic receptors play in SCS-induced peripheral vasodilatation, Linderoth et al (34) found that the vasodilatory effects of SCS were inhibited by beta and ganglionic blockers, but not muscarinic antagonists. They concluded that the SCS-induced inhibitory effect on peripheral vasoconstriction was maintained via efferent sympathetic activity involving pre- and postganglion adrenoreceptors. Increases in sympathetic tone can also result in increased renal arterial pressure (35).

Clinical studies have demonstrated a relative increase in parasympathetic activity affecting visceral organs with spinal cord stimulation. This is demonstrated by the effects of sacral nerve stimulation, a procedure used to decrease bladder hyperactivity and increase bladder capacity. A contributing factor to the beneficial effect SCS exerts on ischemic cardiac muscles has been postulated to be an increase in vagal nerve output (36). Thakkar et al (37) reported the development of a plethora of different gastrointestinal symptoms presumably due to unopposed parasympathetic activity in 2 patients who underwent SCS implantation. The adverse effects that developed were severe enough to require cessation of therapy despite excellent analgesia.

In the normal state, cardiovascular responses to decreased renal blood flow are regulated by a reflex mechanism involving the sympathetic chain at the T9 to L4 levels. This reflex response leads to an elevation in mean arterial pressure and heart rate, which in turn promote increases in renal blood flow and glomerular filtration rate (GFR) (38).

In theory, the decreased sympathetic input induced by SCS could potentially lead to diminished renal blood flow via peripheral shunting, decreased renal perfusion pressure, and an attenuated cardiovascular response to the ensuing hypotension.

There are several proposed mechanisms for the peripheral vasodilatory effects of spinal cord stimulation. These include antidromic activation of sensory fibers of the vanilloid receptor-1 type (39), increased local release of calcitonin gene related peptide (40), local release of nitric oxide (41), and activation of extracellular signal-regulated kinase (ERK) and protein kinase $B(A K T)$ (42). Despite emerging information regarding these effects, they generally apply to cutaneous vasodilation and hence do not address the effect SCS has on perfusion to other organs and tissues. Exactly how much sympathetic inhibition contributes to hypotension remains unknown. The lone study that addressed the effects SCS has on mean arterial pressures failed to detect a difference in normal volunteers (43). In the present case, we operated on the premise that SCS may have unmasked developing ARF that developed primarily in response to the patient's pre-existing condition and superimposed volume depletion.

Although one cannot rule out coincidence, the timing of the occurrence makes it conceivable that our patient's ARF developed from a confluence of events concluding with SCS. These events include a history of hypertension, the medications he was taking, preoperative hypovolemia exacerbated by NPO status, with further reductions in renal blood flow from the advent of SCS. The postoperative hypotension was probably present on POD \#0 as evidenced by the patient's syncopal episode, and persisted for 2 additional days until treatment in the emergency room.

So what can one conclude from this report? We do not believe that hypertension or even preexisting nephropathy should be a contraindication to SCS. Rather, this case at the very least opens up the discussion on the possible role SCS plays in altering the function of other organs. We believe that SCS should be implemented with caution in patients with renal disease. A more detailed history and physical may reveal developing illnesses or hypovolemia. Some possible steps that may reduce even further the remote risk of renal failure in patients having SCS include obtaining pre- and postoperative electrolytes in patients at risk for kidney disease, maintaining adequate pre- and post-implantation hydration, and alerting patients to seek prompt medical evaluation should they develop any untoward symptoms. 


\section{Conclusion}

In conclusion, we report a case of ARF in a patient with hypertension during a spinal cord stimulator trial. The development of the episode of ARF could have been affected, in part, by the secondary effects of SCS. Since ARF has never been associated with the use of SCS, this singular example does not by itself demonstrate a relationship. However, if future episodes are seen, a link between the 2 events could be drawn. For now, it is not clear if this case could be attributed to the use of the spinal cord stimulator or if it was coincidental in nature. We do feel it is useful for the clinician to understand the pathophysiologic changes associated with SCS and to see how, at least in theory, there could be a connection.

\section{References}

1. Melzack R, Wall PD. Pain mechanisms: A new theory. Science 1965; 150:971979.

2. Shealy CN, Mortimer JT, Res wick JB. Electrical inhibition of pain by stimulation of the dorsal columns: Preliminary clinical report. Anesth Analg 1967; 46:489-491.

3. Yakhnitsa V, Linderoth B, Meyerson BA Spinal cord stimulation attenuates dorsal horn neuronal hyperexcitability in a rat model of mononeuropathy. Pain. 1999; 79:223-233.

4. Dubuisson D. Effect of dorsal-column stimulation on gelatinosa and marginal neurons of cat spinal cord. J Neurosurg. 1989; 70:257-265.

5. Meyerson BA, Linderoth B. Mechanisms of spinal cord stimulation in neuropathic pain. Neurol Res 2000; 22:285-292.

6. Tonelli L, Setti T, Falasca A ,Martignoni E, Torcia E, Calcaterra FM, Merli GA, Facchinetti F. Investigation on cerebrospinal fluid opioids and neurotransmitters related to spinal cord stimulation. Appl Neurophysiol 1988; 51:324-332.

7. Meglio M. Spinal cord stimulation in chronic pain management. Neurosurg Clin N Am 2004; 15:297306.

8. Ubbink DT, Vermeulen H, Spincemaille GH, Gersbach PA, Berg P, Amann W. Systematic review and meta-analysis of controlled trials assessing spinal cord stimulation for inoperable critical leg ischaemia. Br J Surg. 2004; 91:948-955.

9. DeJongste MJ. Spinal cord stimu lation for ischemic heart disease. Neurol Res 2000; 22:293-298.

10. Ekre O, Eliasson T, Norrsell H, Wahrborg $\mathrm{P}$, Mannheimer C. Electrical stimulation versus coronary artery bypass surgery in severe angina pectoris. Long-term effects of spinal cord stimulation and coronary artery bypass grafting on quality of life and survival in the ESBY study. Eur Heart J 2002; 23:1938-1945.

11. Sundaraj SR, Johnstone C, Noore F, Wynn P, Castro M. Spinal cord stimulation: A seven-year audit. J Clin Neurosci 2005; 12:264-270.

12. Long DM, Erickson DE. Stimulation of the posterior columns of the spinal cord for relief of intractable pain. Surg Neurol 1975; 4:134-141.

13. Sweet WH. Control of pain by direct electrical stimulation of peripheral nerves. Clin Neurosurg. 1976; 23:103111

14. Kupers RC, Van den Oever R, Van Houdenhove B, Vanmechelen W, Hepp B, Nuttin B, Gybels JM. Spinal cord stimulation in Belgium: A nation-wide survey on the incidence, indications and therapeutic efficacy by the health insurer. Pain 1994; 56:211-216.

15. Turner JA, Loeser JD, Deyo RA, Sanders SB. Spinal cord stimulation for patients with failed back surgery syndrome or complex regional pain syndrome: $A$ systematic review of effectiveness and complications. Pain 2004; 108:137147.

16. Van Buyten JP, Zundert JV, Milbouw G. Treatment of failed back surgery syndrome patients with low back and leg pain: a pilot study of a new dual lead spinal cord stimulation system. Neuromodulation 1999; 2:258-256.

17. Burton AW, Fukshansky M, Brown J, Hassenbusch SJ. Refractory Insomnia in a Patient with Spinal Cord Stimula- tor Lead Migration Neuromodulation 2004; 7: 242-245.

18. Kumar K, Malik S, Demeria D. Treatment of chronic pain with spinal cord stimulation versus alternative therapies: cost-effectiveness analysis. Neurosurgery 2002; 51:106-115.

19. Murphy PM, MacSullivan R. Spinal Cord stimulation for refractory angina pectoris: A shocking experience. Neuromodulation 2004; 7:246.

20. Rothbard RL, Schreiner BF, Yu PN. Hemodynamic and respiratory effects of dezocine, ciramadol, and morphine Clin Pharmacol Ther 1985; 38:84-88.

21. Camu F, Van Overberge L, Bullingham R, Lloyd J. Hemodynamic effects of two intravenous doses of ketorolac tromethamine compared with morphine. Pharmacotherapy 1990; 10:122S-126S.

22. Popio KA, Jackson DH, Ross AM, Schreiner BF, Yu PN. Hemodynamic and respiratory effects of morphine and butorphanol. Clin Pharmacol Ther 1978; 23:281-287.

23. Andersen NH, Morgensen CE. Dual blockade of the renin angiotensin system in diabetic and nondiabetic kidney disease. Curr Hypertens Rep 2004; 6: 369-376.

24. Macias-Nunez JF, Fernandez R, Calvo C, Grande J, Herrera J, Bustamante J, Garay $R$, Robles R, Lopez-Novoa JM. Verapamil reverts acute renal functional impairment induced by angiotensin II converting enzyme inhibitors. Ren Fail 2003; 25:727-737.

25. Prakash J, Murthy AS, Vohra R, Rajak M, Mathur SK. Acute renal failure in the intensive care unit. J Assoc Physicians India 2006; 54:784-788. 
26. W. Gordon Walker and Andrew Welton. Oliguria and renal failure. In Harvey AM, Johns RJ, McKusick VA, Owens AH, Ross RS (Eds). The Principle and Practice of Medicine. Appleton and Lange. Norwalk, CT, 1988. pp.756-76o.

27. Wu M, Linderoth B, Foreman RD. Putative mechanisms behind effects of spinal cord stimulation on vascular diseases: A review of experimental studies. Auton Neurosci 2008; 138:9-23.

28. Sagher O, Huang DL. Mechanisms of spinal cord stimulation in ischemia. Neurosurg Focus 2006; 21:E2.

29. Tanaka S, Komori N, Barron KW, Chandler MJ, Linderoth B, Foreman RD. Mechanisms of sustained cutaneous vasodilation induced by spinal cord stimulation. Auton Neurosci 2004; 114:55-60.

30. Tanaka S, Barron KW, Chandler MJ, Linderoth B, Foreman RD. Role of primary afferents in spinal cord stimulationinduced vasodilation: Characterization of fiber types. Brain Res 2003; 959:191198.

31. Sagher O, Huang DL. Effects of cervical spinal cord stimulation on cerebral blood flow in the rat. I Neurosurg 2000; 93(1 Suppl):71-76.

32. Augustinsson LE, Augustinsson CA, Holm CJ, Jivegard L. Epidural electri- cal stimulation in severe limb ischemia. Pain relief, increased blood flow, and a possible limb-saving effect. Ann Surg 1985; 202:104-110.

33. Linderoth B, Fedorcsak I, Meyerson BA. Peripheral vasodilatation after spinal cord stimulation: animal studies of putative effector mechanisms, Neurosurgery 1991; 28:187-195.

34. Linderoth B, Herregodts P, Meyerson BA. Sympathetic mediation of peripheral mediation of peripheral vasodilation induced by spinal cord stimulation: animal studies of the role of cholinergic and adrenergic receptor subtypes. $\mathrm{Neu}$ rosurgery 1994; 35:711-719.

35. Gao SA, Ambring A, Lambert G, Karlsson AK. Autonomic control of the heart and renal vascular bed during autonomic dysreflexia in high spinal cord injury. Clin Auton Res 2002; 12:457-464.

36. Olgin JE, Takahashi T, Wilson E, Vereckei A, Steinberg H, Zipes DP. Effects of thoracic spinal cord stimulation on cardiac autonomic regulation of the sinus and atrioventricular nodes. J Cardiovasc Electrophysiol 2002; 13:475-481.

37. Thakkar N, Connelly NR, Vieira P. Gastrointestinal symptoms secondary to implanted spinal cord stimulators. Anesth Analg 2003; 97:547-549.

38. Stella A. The kidney as a sensor: func- tional evidence. J Hypertens Suppl. 1992; 10:S113-S119.

39. Wu M, Komori N, Qin C, Farber JP, Linderoth B, Foreman RD. Sensory fibers containing vanilloid receptor-1 (VR1) mediate spinal cord stimulation-induced vasodilation. Brain Res 2006; 1107:177-184. Epub 2006 Jul 11.

40. Croom JE, Foreman RD, Chandler MJ, Barron KW. Cutaneous vasodilation during dorsal column stimulation is mediated by dorsal roots and CGRP. Am J Physiol 1997; 272(2 Pt 2):H950957.

41. Croom JE, Foreman RD, Chandler MJ, Koss MC, Barron KW. Role of nitric oxide in cutaneous blood flow increases in the rat hindpaw during dorsal column stimulation. Neurosurgery 1997; 40:565-570.

42. Wu M, Komori N, Qin C, Farber JP, Linderoth B, Foreman RD. Extracellular signal-regulated kinase (ERK) and protein kinase $B(A K T)$ pathways involved in spinal cord stimulation (SCS)-induced vasodilation. Brain Res 2008; Jan 12).

43. Schultz DM, Musley S, Beltrand P, Christensen J, Euler D, Warman E. Acute cardiovascular effects of epidural spinal cord stimulation. Pain Physician 2007; 10:677-685. 